Portland State University

PDXScholar

\title{
Multigrid Convergence for Second Order Elliptic Problems with Smooth Complex Coefficients
}

Jay Gopalakrishnan

Portland State University, gjay@pdx.edu

Joseph E. Pasciak

Texas A \& M University - College Station

Follow this and additional works at: https://pdxscholar.library.pdx.edu/mth_fac

Part of the Analysis Commons, and the Applied Mathematics Commons Let us know how access to this document benefits you.

\section{Citation Details}

Gopalakrishnan, Jay and Pasciak, Joseph E., "Multigrid Convergence for Second Order Elliptic Problems with Smooth Complex Coefficients" (2008). Mathematics and Statistics Faculty Publications and Presentations. 60.

https://pdxscholar.library.pdx.edu/mth_fac/60

This Post-Print is brought to you for free and open access. It has been accepted for inclusion in Mathematics and Statistics Faculty Publications and Presentations by an authorized administrator of PDXScholar. Please contact us if we can make this document more accessible: pdxscholar@pdx.edu. 


\title{
MULTIGRID CONVERGENCE FOR SECOND ORDER ELLIPTIC PROBLEMS WITH SMOOTH COMPLEX COEFFICIENTS
}

\author{
JAYADEEP GOPALAKRISHNAN AND JOSEPH E. PASCIAK
}

\begin{abstract}
The finite element method when applied to a second order partial differential equation in divergence form can generate operators that are neither Hermitian nor definite when the coefficient function is complex valued. For such problems, under a uniqueness assumption, we prove the continuous dependence of the exact solution and its finite element approximations on data provided that the coefficients are smooth and uniformly bounded away from zero. Then we show that a multigrid algorithm converges once the coarse mesh size is smaller than some fixed number, providing an efficient solver for computing discrete approximations. Numerical experiments, while confirming the theory, also reveal pronounced sensitivity of Gauss-Seidel iterations on the ordering of the unknowns for certain problems.
\end{abstract}

\section{InTRODUCTION}

We consider the application of multigrid algorithms to second order partial differential equations whose dominant coefficient is complex valued. In particular, we have in mind complex valued coefficients generating operators that may not be Hermitian or definite. A study of problems with such coefficients is the first step towards understanding the behavior of multigrid applied to important problems with complex coefficients such as those arising from time harmonic scattering and radiation. In particular, applications of the perfectly matched layer technique (PML) to scattering problems and resonance problems lead to complex coefficient problems, however, in this case, the problem is no longer in divergence form and exhibits anisotropic behavior $[4,5,13]$. The study of multigrid applied to the PML problem is a topic for future research.

Let $\Omega$ be a polygonal domain in $\mathbb{R}^{2}$. We allow $\Omega$ to be non-convex but require that its boundary be Lipschitz continuous. As a model problem, we consider

$$
\begin{aligned}
-\nabla \cdot \alpha(x) \nabla u & =f \text { in } \Omega, \\
u & =0 \text { on } \partial \Omega,
\end{aligned}
$$

where $\alpha$ is a complex valued non-vanishing function defined on $\Omega$. In the above equation and the rest of this paper, the dot denotes the dot product without complex conjugation, i.e., for vectors $v=\left(v_{1}, v_{2}\right)$ and $w=\left(w_{1}, w_{2}\right)$, we set $v \cdot w=v_{1} w_{1}+v_{2} w_{2}$. A variational formulation of (1.2) is posed on the Sobolev space $H_{0}^{1}(\Omega)$ consisting of those complex valued functions vanishing on $\partial \Omega$ which, along with their first derivatives, are in $L^{2}(\Omega)$ (the space of complex valued functions whose absolute values are square integrable).

2000 Mathematics Subject Classification. 65F10, 65M55, 65N55, 65N30, 74G15.

Key words and phrases. multigrid, nonsymmetry, complex, finite element, V-cycle, backslash cycle, Gauss-Seidel, ordering, smoothing, perturbation, preconditioning.

This work was supported in part by the National Science Foundation (USA) under grants DMS0713833, SCREMS-0619080, and DMS-0609544. 
We seek $u \in H_{0}^{1}(\Omega)$ satisfying

$$
a(u, \phi)=(f, \phi) \quad \text { for all } \phi \in H_{0}^{1}(\Omega) .
$$

Here

and

$$
a(u, v) \equiv \int_{\Omega} \alpha \nabla u \cdot \nabla \bar{v} d x
$$

$$
(f, g) \equiv \int_{\Omega} f \bar{g} d x
$$

$(\bar{v}$ denotes the complex conjugate of $v$ ). Of course, $(\cdot, \cdot)$ is just the Hermitian inner product on $L^{2}(\Omega)$ and we shall denote the corresponding norm by $\|\cdot\|$.

We shall assume uniqueness for (1.2), i.e., if $v \in H_{0}^{1}(\Omega)$ satisfies

$$
a(v, \phi)=0 \quad \text { for all } \phi \in H_{0}^{1}(\Omega)
$$

then $v=0$. It is not obvious when (1.3) holds in the case of general $\alpha$. However a class of coefficients for which it is immediately verified is given next.

Example 1.1. Assumption (1.3) holds if there is a complex number $\beta_{0}$ and positive constant $c_{0}$ satisfying

$$
c_{0} \leq \operatorname{Re}\left(\beta_{0} \alpha(x)\right) \text { for all } x \in \Omega .
$$

This condition implies a coercivity inequality of the form

$$
|w|_{H^{1}(\Omega)}^{2} \leq\left|a\left(w, \bar{\beta}_{0} w\right)\right| \quad \text { for all } w \in H_{0}^{1}(\Omega)
$$

from which uniqueness immediately follows. Here $|\cdot|_{H^{1}(\Omega)}$ denotes the $H^{1}(\Omega)$-seminorm. Thus there are many complex coefficient problems satisfying our assumption.

Under Assumption (1.3), we shall show that the solution of problem (1.2) exists. By using a scaled test function, the problem (1.2) can be related to a coercive problem with a low order perturbation and classical arguments along the lines of Peetre [15] and Tartar [17] can be applied. This perturbation approach will be carried out at the discrete level as well to show that the discrete problem has solutions for sufficiently fine meshes.

The stability of the discrete problem for fine enough $h$ is the starting point of the construction and analysis of the multigrid algorithm. The multigrid algorithm that we shall consider is variational, i.e., built with nested spaces and inherited forms (see, e.g., [7]). Our analysis is based on perturbation arguments. Perturbation arguments have been widely applied for the analysis of multigrid algorithms corresponding to coercive differential operators perturbed by lower order terms [3, $7,11,18]$. While we continue to rely on the basic perturbation idea, our point of departure in this paper is that we perturb the dominant (highest order) term in the differential equation. It is interesting that multigrid perturbation techniques can be made to work for this application where the perturbation is not of low order.

We prove that once the coarse mesh size (in the multigrid algorithm) is smaller than a fixed number depending on $\alpha$, a standard multigrid algorithm converges at a mesh independent rate. This implies that as the mesh size goes to zero, the number of iterations needed to reduce the initial error by a fixed tolerance factor is asymptotically bounded by a fixed number $C_{1}$. If $N$ is the number of unknowns, and if the number of flops required for one coarse solve is at most $C_{0}$, then the cost of one iteration is $O(N)+C_{0}$. Hence the total cost before meeting the stopping criterion is $C_{1}\left(O(N)+C_{0}\right)$. Thus, our theoretical 
result implies that the algorithm yields a solver of asymptotically optimal complexity. Of course both $C_{0}$ and $C_{1}$ can depend on the coefficient $\alpha$. It is well known that the performance of many multigrid algorithms deteriorates for difficult coefficients.

Our theory applies to multigrid algorithms utilizing classical point Jacobi or GaussSeidel smoothers. In contrast to the symmetric and positive definite case, these smoothers may, in fact, be mildly unstable (i.e., their error reducing operators may be expansive). It is known that multigrid smoothers can be mildly unstable [1, 3], even for problems with real coefficients when they have lower order indefinite terms. The instability appears to be more pronounced in the case of Gauss-Seidel smoothing for certain nodal orderings. In such cases, a smaller coarse mesh size plays a critical role. This is clearly illustrated in our numerical experiments where finer coarse mesh sizes need to be used for Gauss-Seidel smoothing and lexicographical ordering, while larger coarser meshes work with GaussSeidel smoothers utilizing red/black node ordering or the Jacobi smoother. Clearly, it is of practical importance to be aware of strong dependence of algorithms on the nodal orderings. Another finding of practical importance from this work is the necessity of a fine enough coarse mesh for a standard multigrid algorithm applied to certain complex coefficient problems. While this behavior for the stationary wave equation is well known, it seems to be less known that it can arise for complex coefficient problems as well.

The outline of the remainder of the paper is as follows. In Section 2, we show the stability of (1.2) and its finite element approximation on sufficiently fine meshes. Section 3 introduces the multigrid algorithm, including the definition of the Jacobi and Gauss-Seidel smoothers in a notation which is appropriate for our subsequent analysis. The convergence analysis of the multigrid algorithm is given in Section 4. Finally, the results of numerical experiments are given in Section 5.

\section{Stability and Finite Element approximation}

First, let us establish the continuous dependence of the solution $u$ on the data $f$. Then we will establish a similar result for a discrete approximation.

Along with our uniqueness assumption (1.3), we shall require that our coefficients are smooth and bounded away from zero in absolute value, specifically, we assume that $\alpha$ : $\Omega \mapsto \mathbb{C}$ is in $C^{2}(\bar{\Omega})$ and that there is constant $\alpha_{0}>0$ such that

$$
\alpha_{0} \leq|\alpha(x)| \text { for all } x \in \Omega \text {. }
$$

This assumption implies that the characteristic variety of the partial differential operator is empty and consequently the operator is elliptic [10, p. 33]. Together with problem (1.2), we shall consider the adjoint problem: Find $v \in H_{0}^{1}(\Omega)$ satisfying

$$
a(\phi, v)=(\phi, g) \quad \text { for all } \phi \in H_{0}^{1}(\Omega) .
$$

From now on, we will tacitly assume that (1.3) and (2.1) hold. The next result gives a few consequences of these assumptions.

Proposition 2.1. Suppose that (1.3) and (2.1) hold. Then the following existence and regularity results are valid:

(1) There is a unique $u$ in $H_{0}^{1}(\Omega)$ solving problem (1.2).

(2) There is a unique $v$ in $H_{0}^{1}(\Omega)$ solving the adjoint problem (2.2). 
(3) There is an $s>1 / 2$ and a constant $C_{\mathrm{reg}}>0$ such that the solution $v$ of the adjoint problem $(2.2)$ is in $H^{1+s}(\Omega)$ and satisfies

$$
\|v\|_{H^{1+s}(\Omega)} \leq C_{\mathrm{reg}}\|g\| .
$$

Proof. We first note that we have uniqueness for the adjoint problem as well. Indeed if $v \in H_{0}^{1}(\Omega)$ satisfies

$$
a(\phi, v)=0 \quad \text { for all } \phi \in H_{0}^{1}(\Omega)
$$

then taking $\phi=\bar{w}$ gives

$$
a(\bar{v}, w)=0 \quad \text { for all } w \in H_{0}^{1}(\Omega) .
$$

The uniqueness property (1.3) then implies that $\bar{v}=0$ and hence $v=0$.

To prove items (1) and (2), we start from the following identity:

$$
\int_{\Omega}|\alpha|^{2}|\nabla v|^{2} d x=a(v, \alpha v)-\int_{\Omega} \alpha(\nabla v \cdot \overline{\nabla \alpha}) \bar{v} d x
$$

which holds for any $v \in H_{0}^{1}(\Omega)$. Setting $z=\alpha v$, we then have

$$
\begin{aligned}
\int_{\Omega}|\alpha|^{2}|\nabla v|^{2} d x & =a(v, z)-(\alpha \nabla(z / \alpha) \cdot \overline{\nabla \alpha}, v) \\
& \leq|\alpha v|_{H^{1}(\Omega)}\left(\frac{|a(v, z)|+|(\alpha \nabla(z / \alpha) \cdot \overline{\nabla \alpha}, v)|}{|z|_{H^{1}(\Omega)}}\right) \\
& \leq C\|v\|_{H^{1}(\Omega)}\left(\sup _{w \in H_{0}^{1}(\Omega)} \frac{|a(v, w)|}{\|w\|_{H^{1}(\Omega)}}+\|v\|\right) .
\end{aligned}
$$

Here we have used the smoothness assumption on $\alpha$ and (2.1) for the last inequality. Note that here and elsewhere in this paper we will use the letter $C$ with or without subscripts to denote a generic constant whose value may differ at different occurrences. These constants may depend on $\alpha$ and $\Omega$ but will always remain independent of the meshes and functions involved.

Now, using the nondegeneracy assumption,

$$
|v|_{H^{1}(\Omega)}^{2} \leq \alpha_{0}^{-2} \int_{\Omega}|\alpha|^{2}|\nabla v|^{2} d x
$$

which when combined with the previous inequality and the Poincaré inequality shows that

$$
\|v\|_{H^{1}(\Omega)} \leq C\left(\sup _{w \in H_{0}^{1}(\Omega)} \frac{|a(v, w)|}{\|w\|_{H^{1}(\Omega)}}+\|v\|\right),
$$

for all $v$ in $H_{0}^{1}(\Omega)$.

Next, we apply a well known perturbation argument due to Peetre [15] and Tartar [17]. This uses the compact imbedding of $H^{1}(\Omega)$ into $L^{2}(\Omega)$ and an argument by contradiction to show that the uniqueness assumption (1.3) and (2.4) imply that (2.4) holds without the lower order term, i.e.,

$$
\|v\|_{H^{1}(\Omega)} \leq C \sup _{w \in H_{0}^{1}(\Omega)} \frac{|a(v, w)|}{\|w\|_{H^{1}(\Omega)}} .
$$


The adjoint inf-sup condition

$$
\|w\|_{H^{1}(\Omega)} \leq C \sup _{v \in H_{0}^{1}(\Omega)} \frac{|a(v, w)|}{\|v\|_{H^{1}(\Omega)}}
$$

follows from similar arguments and uniqueness for the adjoint problem (proved above). The above two inf-sup conditions guarantee [9, Ch. II, p. 39] the existence of a (unique) solution to (1.2) as well as the adjoint problem (2.2). This proves items (1) and (2) of the proposition.

For Part (3) of the proposition, note that the solution of the adjoint problem satisfies

$$
-\bar{\alpha} \Delta \psi=g+\overline{\nabla \alpha} \cdot \nabla \psi
$$

hence the required regularity follows from the well known regularity of Laplace solutions on polygonal domains $[12,14]$.

Next, we describe the finite element approximation of the exact solution $u$ of problem (1.2). Let $\mathcal{T}_{h}$ denote a quasiuniform triangulation of $\Omega$ (with the usual geometrical conformity assumptions for finite elements). The representative diameters of the mesh elements is denoted by $h$, e.g., $h=\max \left\{\operatorname{diam}(K): K \in \mathcal{T}_{h}\right\}$. The approximation space is

$$
V_{h}=\left\{v \in L^{2}(\Omega): \quad v \text { is continuous, }\left.v\right|_{K} \text { is linear for all } K \in \mathcal{T}_{h} \text {, and }\left.v\right|_{\partial \Omega}=0\right\} .
$$

To guarantee that the finite element method applied to our problem is well defined, we must check that there is a unique $u_{h}$ in $V_{h}$ satisfying

$$
a\left(u_{h}, v_{h}\right)=\left(f, v_{h}\right), \quad \text { for all } v_{h} \in V_{h} .
$$

This will follow as a consequence of the next lemma. The estimate of the lemma is the discrete analogue of (2.4).

Lemma 2.1. There is a positive number $h_{0}$ such that if $h \leq h_{0}$,

$$
\|v\|_{H^{1}(\Omega)} \leq C\left(\sup _{w \in V_{h}} \frac{|a(v, w)|}{\|w\|_{H^{1}(\Omega)}}+\|v\|\right) \quad \text { for all } v \in V_{h} .
$$

Proof. Since $\alpha \in C^{2}(\bar{\Omega}), \alpha v$ is in $H^{2}(\tau)$ for any triangle $\tau \in \mathcal{T}_{h}$ and any $v$ in $V_{h}$. Moreover, since $\left.v\right|_{\tau}$ is linear,

$$
\|\alpha v\|_{H^{2}(\tau)} \leq C\|v\|_{H^{1}(\tau)}\|\alpha\|_{W_{\infty}^{2}(\tau)} \leq C\|v\|_{H^{1}(\tau)}
$$

It follows that

$$
\left\|\alpha v-I_{h}(\alpha v)\right\|_{H^{1}(\tau)} \leq C h\|v\|_{H^{1}(\tau)}
$$

where $I_{h}$ denotes the nodal interpolation operator associated with $V_{h}$. In addition, by our assumptions on $\alpha$, multiplication by $\alpha$ is a bounded map of $H^{1}(\Omega)$ onto $H^{1}(\Omega)$ and so (2.10) implies that

$$
\left\|I_{h}(\alpha v)\right\|_{H^{1}(\tau)} \leq C\|v\|_{H^{1}(\tau)} .
$$

We will use these properties of the nodal interpolant to prove the lemma. 
By (2.3), for $v \in V_{h}$,

$$
\begin{aligned}
\|v\|_{H^{1}(\Omega)}^{2} \leq & \alpha_{0}^{-2} \int_{\Omega}|\alpha|^{2}|\nabla v|^{2} d x \\
= & \alpha_{0}^{-2}\left(a\left(v, I_{h}(\alpha v)\right)+\int_{\Omega} \alpha \nabla v \cdot\left(\overline{\nabla(\alpha v)-\nabla I_{h}(\alpha v)}\right) d x\right. \\
& \left.\quad-\int_{\Omega} \alpha(\nabla v \cdot \overline{\nabla \alpha}) \bar{v} d x .\right) .
\end{aligned}
$$

Applying (2.10) and the Cauchy-Schwarz inequality, we obtain

$$
\|v\|_{H^{1}(\Omega)} \leq C\left(\frac{\left|a\left(v, I_{h}(\alpha v)\right)\right|}{\|v\|_{H^{1}(\Omega)}}+h\|v\|_{H^{1}(\Omega)}+\|v\|\right) .
$$

Thus whenever $h$ is so small that $1-C h>0$, we have

$$
\begin{aligned}
(1-C h)\|v\|_{H^{1}(\Omega)} & \leq C\left(\frac{\left|a\left(v, I_{h}(\alpha v)\right)\right|}{\|v\|_{H^{1}(\Omega)}}+\|v\|\right) \\
& \leq C\left(\frac{\left|a\left(v, I_{h}(\alpha v)\right)\right|}{\left\|I_{h}(\alpha v)\right\|_{H^{1}(\Omega)}}+\|v\|\right),
\end{aligned}
$$

where we used (2.11). The lemma now follows from (2.13) by taking the supremum.

We can now address the solvability of finite element approximations and, in particular, the coarse grid problem for multigrid. Specifically, we consider the problem: Given $v \in$ $V \equiv H_{0}^{1}(\Omega)$, find $v_{h} \in V_{h}$ satisfying

$$
a\left(v_{h}, \theta\right)=a(v, \theta) \quad \text { for all } \theta \in V_{h} .
$$

The next theorem guarantees unique solvability for sufficiently small $h$.

Theorem 2.1. There is an $h_{0}>0$ such that for $h \leq h_{0}$, there is a unique solution $v_{h}$ to (2.14) for any $v \in V$ and

$$
\left\|v_{h}\right\|_{H^{1}(\Omega)} \leq C\|v\|_{H^{1}(\Omega)} .
$$

Proof. We follow the duality approach of Schatz [16]. If $v_{h}$ is any solution to (2.14) then $e_{h}=v-v_{h}$ satisfies the Galerkin orthogonality equation

$$
a\left(e_{h}, w_{h}\right)=0 \text { for all } w_{h} \in V_{h} .
$$

By Proposition 2.1(2), there is a unique $\varepsilon$ in $H_{0}^{1}(\Omega)$ satisfying $a(w, \varepsilon)=\left(w, e_{h}\right)$ for all $w$ in $H_{0}^{1}(\Omega)$. Hence

$$
\begin{aligned}
\left\|e_{h}\right\|^{2} & =a\left(e_{h}, \varepsilon\right)=a\left(e_{h}, \varepsilon-I_{h} \varepsilon\right), \\
& \leq C h^{s}\|\varepsilon\|_{H^{1+s}(\Omega)}\left\|e_{h}\right\|_{H^{1}(\Omega)} .
\end{aligned}
$$

Applying Proposition 2.1(3), we find that the estimate

$$
\left\|v-v_{h}\right\| \leq C h^{s}\left\|v-v_{h}\right\|_{H^{1}(\Omega)}
$$

holds whenever $v_{h}$ satisfies (2.14).

Now, suppose that $v_{h}$ satisfies (2.14) with $v=0$. Lemma 2.1 applied to $v_{h}$ and (2.17) gives

$$
\left\|v_{h}\right\|_{H^{1}(\Omega)} \leq C\left\|v_{h}\right\| \leq C h^{s}\left\|v_{h}\right\|_{H^{1}(\Omega)}
$$


for sufficiently small $h$. It follows that $v_{h}$ must vanish for sufficiently small $h$. This means that the only solution of (the square system) (2.14) is $v_{h} \equiv 0$. Existence and uniqueness of the solutions to (2.14) for such $h$ follow for nonzero $v$.

Finally, to prove the estimate (2.15), observe that Lemma 2.1 and (2.17) give

$$
\begin{aligned}
\left\|v_{h}\right\|_{H^{1}(\Omega)} & \leq C\left(\sup _{w \in V_{h}} \frac{\left|a\left(v_{h}, w\right)\right|}{\|w\|_{H^{1}(\Omega)}}+\left\|v_{h}\right\|\right)=C\left(\sup _{w \in V_{h}} \frac{|a(v, w)|}{\|w\|_{H^{1}(\Omega)}}+\left\|v_{h}\right\|\right) \\
& \leq C\left(\|v\|_{H^{1}(\Omega)}+\left\|v-v_{h}\right\|+\|v\|\right) \leq C\left(\|v\|_{H^{1}(\Omega)}+h^{s}\left\|v_{h}\right\|_{H^{1}(\Omega)}\right) .
\end{aligned}
$$

The stability inequality (2.15) follows taking $h$ sufficiently small.

Remark 2.1. By virtue of Theorem 2.1, once the mesh size $h$ is small enough for $v_{h}$ to exist, we can define the projector $P_{h}: V \rightarrow V_{h}$ by $P_{h} v=v_{h}$ where $v_{h}$ solves (2.14). This projector is an important ingredient of our subsequent multigrid analysis.

Remark 2.2. Theorem 2.1 obviously also implies the unique solvability of the finite element method (2.8) once $h$ is small enough. Moreover, the finite element error is quasioptimal. Indeed, if $u$ solves (1.2) and $u_{h}$ solves (2.8), then for any $w_{h}$ in $V_{h}$, by Lemma 2.1,

$$
\begin{aligned}
\left\|u_{h}-w_{h}\right\|_{H^{1}(\Omega)} & \leq C\left(\sup _{z_{h} \in V_{h}} \frac{\left|a\left(u_{h}-w_{h}, z_{h}\right)\right|}{\left\|z_{h}\right\|_{H^{1}(\Omega)}}+\left\|u_{h}-w_{h}\right\|\right) \\
& \leq C\left(\sup _{z_{h} \in V_{h}} \frac{\left|a\left(u-w_{h}, z_{h}\right)\right|}{\left\|z_{h}\right\|_{H^{1}(\Omega)}}+\left\|u-u_{h}\right\|+\left\|u-w_{h}\right\|\right) \\
& \leq C\left(\left\|u-w_{h}\right\|_{H^{1}(\Omega)}+\left\|u-u_{h}\right\|\right) .
\end{aligned}
$$

Hence, using the triangle inequality and (2.17), we find that for sufficiently small $h$,

$$
\left\|u-u_{h}\right\|_{H^{1}(\Omega)} \leq C \inf _{w_{h} \in V_{h}}\left\|u-w_{h}\right\|_{H^{1}(\Omega)} .
$$

Therefore the discretization error of the finite element method applied to our problem converges to zero in $H^{1}(\Omega)$ at the optimal rate as $h$ tends to zero.

\section{A multigrid ALGORITHM}

The basis for geometrical multigrid algorithms is a coarse grid and a sequence of its refinements. We start with a coarse triangulation of $\Omega$, namely $\mathcal{T}_{1}=\left\{\tau_{1}^{i}: i=1, \ldots N_{0}\right\}$. This coarse mesh size is the basis of our refined grids, but may not be the coarse mesh size used in the multigrid algorithm. As this coarse mesh is fixed, it is quasiuniform with mesh size $h_{1}=\max \left\{\operatorname{diam}(K): K \in \mathcal{T}_{1}\right\}$. A nested sequence of quasiuniform meshes $\mathcal{T}_{k}$, $k=2,3, \ldots$, of mesh size $h_{k}=h_{1} / 2^{k-1}$ is obtained by successively refining $\mathcal{T}_{1}$, namely the mesh $\mathcal{T}_{k}$ is obtained by connecting the midpoints of the edges of $\mathcal{T}_{k-1}$. Let $V_{k}$ be the space obtained by replacing $\mathcal{T}_{h}$ with $\mathcal{T}_{k}$ in (2.7). These spaces form our sequence of nested multilevel spaces.

We want to efficiently solve the Galerkin approximation to (1.2) associated with the finest mesh $\mathcal{T}_{J}$ (or the largest space $V_{J}$ ). Specifically, we want a multigrid scheme to use in an iteration for computing $u_{J}$ in $V_{J}$ solving

$$
a\left(u_{J}, v\right)=(f, v) \text { for all } v \in V_{J} .
$$

Our goal is to study the behavior of the so called "multigrid V-cycle" for this complex coefficient discrete problem. 
Before we state the multigrid algorithm, we need to define "smoothers". Our smoothers are linear maps $R_{k}: V_{k} \rightarrow V_{k}$. We shall start with the map associated with the point Gauss-Seidel iteration. First let $D_{k, i}$ denote the domain formed by all the mesh triangles connected to the $i^{\text {th }}$ vertex $x_{k, i}\left(i=1,2, \ldots, N_{k}\right)$ of the $k^{\text {th }}$ level mesh $\mathcal{T}_{k}$. Let $V_{k, i}$ denote the set of functions in $V_{k}$ which are supported on $D_{k, i}$ (this is just the one dimensional space spanned by the nodal basis function for the subspace $V_{k}$ at the node $x_{k, i}$ ). Define $A_{k, i}: V_{k, i} \mapsto V_{k, i}$, by

$$
\left(A_{k, i} w, \phi\right)=a(w, \phi), \quad \text { for all } \phi, w \in V_{k, i} .
$$

Algorithm 3.1 (Point Gauss Seidel smoother). For any $b$ in $V_{k}$, we define $G_{k} b$ as follows. First, we set $v_{0}$ to be the zero function in $V_{k}$ and set $G_{k} b=v_{N_{k}}$ where for $i=1,2, \ldots, N_{k}$,

$$
v_{i}=v_{i-1}+\left(A_{k, i}\right)^{-1} Q_{k, i}\left(b-A_{k} v_{i-1}\right) .
$$

The above algorithm is just the approximate inverse corresponding to the classical Gauss-Seidel iterative method in disguise. We present it in terms of subspaces since it is more convenient for our subsequent analysis. We shall also consider the point GaussSeidel smoother which results from visiting the above subspaces in reverse order, which we denote by $G_{k}^{\prime}$. Another standard smoother that can be used in our multigrid algorithm is the classical Jacobi smoother, which is the additive version of the above algorithm. It can be written as

$$
J_{k} v=\beta \sum_{i=1}^{N_{k}}\left(A_{k, i}\right)^{-1} Q_{k, i}\left(b-A_{k} v\right) .
$$

The constant $\beta$ is a damping parameter.

We require some additional notation to define the multigrid algorithm. Specifically, we define $A_{k}: V_{k} \mapsto V_{k}$ by

$$
\left(A_{k} w, \phi\right)=a(w, \phi)
$$

for all $\phi$ and $w$ in $V_{k}$ and set $Q_{k}$ to be the $L^{2}(\Omega)$ orthogonal projection onto $V_{k}$. Finally, we introduce an integer $k_{0} \in\{1,2, \ldots, J-1\}$ which sets the coarse grid size for the multigrid algorithm. Then the V-cycle multigrid algorithm is defined inductively and is denoted by $\operatorname{MG}_{k}(v, w)$ where $k$ is the level number and $v, w$ are in $V_{k}$. The smoother $R_{k}$ in the algorithm below can be set to either the Gauss-Seidel smoother $G_{k}$ or the Jacobi smoother $J_{k}$ and $R_{k}^{\prime}$ can be set to either $G_{k}^{\prime}$ or $J_{k}$.

Algorithm 3.2 (V-cycle). Set $\mathrm{MG}_{k_{0}}(v, w)=A_{k_{0}}^{-1} w$. Let $k>k_{0}$ and $v, w \in V_{k}$. Assuming that $\mathrm{MG}_{k-1}(\cdot, \cdot)$ has been defined, we define $\mathrm{MG}_{k}(v, w)$ as follows:

(1) Set $x=v+R_{k}\left(w-A_{k} v\right)$.

(2) Set $y=x+\mathrm{MG}_{k-1}\left(0, Q_{k-1}\left(w-A_{k} x\right)\right)$.

(3) Define $\mathrm{MG}_{k}(v, w)=y+R_{k}^{\prime}\left(w-A_{k} y\right)$.

The multigrid iterative scheme for obtaining the solution $u_{J}$ of (3.1) is as follows. With some initial iterate $u_{J}^{(0)}$, we define a sequence of further iterates by

$$
u_{J}^{(i+1)}=\mathrm{MG}_{J}\left(u_{J}^{(i)}, Q_{J} f\right), \quad i=1,2, \ldots
$$

Our analysis in the next section will show that once $k_{0}$ is larger than some fixed number, the above iterates $u_{J}^{(i)}$ converge to $u_{J}$ as $i$ increases, at a rate independent of $J$.

It is important to note that for Algorithm 3.2 to be well defined, the coarse grid problem and all of the smoothers must be well defined. This can be achieved by taking the coarse 
grid in the multigrid algorithm to be sufficiently fine (i.e., taking $k_{0}$ large enough), as we now show. By making $k_{0}$ large enough, the mesh size $h_{k_{0}}$ becomes small enough to apply Theorem 2.1 with $V_{h}=V_{k_{0}}$. This shows that $A_{k_{0}}$ is invertible whenever $k_{0}$ is large enough. To show that the smoothers are also well defined under the same condition, it suffices to show that the invertibility of $A_{k, i}$, or equivalently

$$
\|v\|_{H^{1}\left(D_{k, i}\right)} \leq C \sup _{w \in V_{k, i}} \frac{|a(v, w)|}{\|w\|_{H^{1}\left(D_{k, i}\right)}} \text { for all } v \in V_{k, i} .
$$

To show this, first observe that an inequality analogous to that of Lemma 2.1 holds for $V_{k, i}$. Indeed, reviewing the proof of Lemma 2.1, we obtain

$$
\|v\|_{H^{1}\left(D_{k, i}\right)} \leq C\left(\sup _{w \in V_{k, i}} \frac{|a(v, w)|}{\|w\|_{H^{1}\left(D_{k, i}\right)}}+\|v\|\right) \quad \text { for all } v \in V_{k, i} .
$$

Since $\operatorname{diam}\left(D_{k, i}\right) \leq C h_{k}$, the Poincaré inequality yields

$$
\|v\| \leq C h_{k}\|v\|_{H^{1}\left(D_{k, i}\right)}, \quad \text { for all } v \in V_{k, i} .
$$

Using this in (3.5), we have

$$
\left(1-C h_{k}\right)\|v\|_{H^{1}\left(D_{k, i}\right)} \leq C \sup _{w \in V_{k, i}} \frac{|a(v, w)|}{\|w\|_{H^{1}\left(D_{k, i}\right)}} \text { for all } v \in V_{k, i},
$$

from which (3.4) follows once $h_{k}$ is small enough.

Finally, note that it is equally appropriate to consider other multigrid algorithms, e.g., those involving smoothing only in step (1) or only in step (3) above. In fact, for simplicity, in the next section we shall analyze the algorithm obtained from Algorithm 3.2 by eliminating step (3), or more precisely, we define another algorithm by replacing step (3) of Algorithm 3.2 by

$$
\mathrm{MG}_{k}^{\backslash}(v, w)=y
$$

The new algorithm, $\mathrm{MG}_{k}^{\backslash}(\cdot, \cdot)$, is often known as the "backslash multigrid cycle" or simply the \-cycle.

\section{Multigrid ANALYSis}

We will now give an analysis of the \-cycle algorithm (involving only pre-smoothing) applied to the sesquilinear form (1.2). The analysis for the "more symmetric" V-cycle algorithm is essentially identical, save more notation.

To begin, observe that $\mathrm{MG}_{J}^{\backslash}(\cdot, \cdot)$ is a linear map from $V_{J} \times V_{J}$ into $V_{J}$. Moreover, it is a consistent iteration in the sense that $v=\mathrm{MG}_{J}\left(v, A_{J} v\right)$ for all $v \in V_{J}$. It easily follows that the linear operator $\mathcal{E} \equiv \mathcal{E}_{J}=\mathrm{MG}_{J}(\cdot, 0)$ is the error reduction operator for (3.3), i.e., if $u_{i+1}=\mathrm{MG}_{J}\left(u_{i}, Q_{J} f\right)$, then

$$
u-u_{i+1}=\mathcal{E}\left(u-u_{i}\right) .
$$

There is a well known [6] product representation for multigrid error reduction operators. Let $T_{k}=R_{k} A_{k} P_{k}$ for $k>k_{0}$ and set $T_{k_{0}}=P_{k_{0}}$. We will assume throughout this section that $h_{k_{0}}<h_{0}$ ( where $h_{0}$ is the number of Theorem 2.1) so that $P_{k}$ is well defined for $k=k_{0}, \ldots, J$. Let $\mathcal{E}_{k} u=u-\mathrm{MG}_{k}\left(0, A_{k} P_{k} u\right)$ and $\mathcal{E}_{k_{0}-1} \equiv I$, the identity operator. Then

$$
\varepsilon_{k}=\mathcal{E}_{k-1}\left(I-T_{k}\right) \quad \text { for } k=k_{0}, \ldots, J
$$


and

$$
\mathcal{E}=\left(I-T_{k_{0}}\right)\left(I-T_{k_{0}+1}\right) \cdots\left(I-T_{J}\right) .
$$

This product representation of the error reducing operator will be the starting point of our convergence analysis.

Suppose we apply the \-cycle version of algorithm (Algorithm 3.2) to solve the standard Laplace's equation, i.e., using $A_{k}, R_{k}$ and $R_{k}^{\prime}$ in the algorithm defined from the bilinear form

$$
\hat{a}(u, v)=(\nabla u, \nabla v) .
$$

Then the identity analogous to identity (4.1) holds for the corresponding operators as this is only a special case of the variable coefficient case. To distinguish it from the general case, we use notations with “^”, i.e., any previously defined notation superscripted with ^ indicates that it is defined as before but by replacing $a(u, v)$ with $\hat{a}(u, v)$. Thus,

$$
\hat{\mathcal{E}}=\left(I-\hat{T}_{k_{0}}\right)\left(I-\hat{T}_{k_{0}+1}\right) \cdots\left(I-\hat{T}_{J}\right),
$$

where $\hat{T}_{k}=\hat{R}_{k} \hat{A}_{k} \hat{P}_{k}$ for $k>k_{0}, \hat{T}_{k_{0}}=\hat{P}_{k_{0}}$, etc.

Our analysis proceeds by a perturbation argument bounding the difference between $\mathcal{E}$ and $\hat{\mathcal{E}}$. To simplify notation, let

$$
\|u\|_{\Lambda}=\hat{a}(u, u)^{1 / 2} .
$$

The operator norm induced by this norm is also denoted by $\|\cdot\|_{\Lambda}$. Let $Z_{k}=T_{k}-\hat{T}_{k}$ and suppose we have

$$
\begin{aligned}
\left\|Z_{k_{0}}\right\|_{\Lambda} & \leq \epsilon, \quad \text { and } \\
\left\|Z_{k}\right\|_{\Lambda} & \leq C_{1} h_{k}, \text { for } k=k_{0}+1, \ldots, J .
\end{aligned}
$$

Then, it can be shown that the difference between the operator norms of $\mathcal{E}_{k}$ and $\hat{\mathcal{E}}_{k}$ is small by an argument of [3] (see also [7, Lemma 11.1] and [11, Theorem 4.2]). We state this result in the following lemma.

Lemma 4.1 (Multigrid perturbation). Let $\mathcal{E}$ satisfy (4.1) and $\hat{\mathcal{E}}$ satisfy (4.2). Assume that (4.3) and (4.4) holds. Then

$$
\|\mathcal{E}-\hat{\mathcal{E}}\|_{\Lambda} \leq C\left(h_{k_{0}}+\epsilon\right) .
$$

The proofs of our main result proceeds by verifying (4.3) and (4.4). We treat the case of Gauss-Seidel smoothing. That of Jacobi smoothing is similar. Define $P_{k, i}: V_{k} \mapsto V_{k, i}$ by

$$
a\left(P_{k, i} u, v_{i}\right)=a\left(u, v_{i}\right) \quad \text { for all } u \in V_{k}, v_{i} \in V_{k, i},
$$

and $\hat{P}_{k, i}$ similarly using the $\hat{a}$-form. Note that while the stability of $\hat{P}_{k, i}$ in $\Lambda$-norm is obvious, the solvability and stability of (4.5) follows from (3.4) for sufficiently small $h_{k}$. Indeed, by (3.4),

$$
\left\|P_{k, i} u\right\|_{\Lambda} \leq C\|\nabla u\|_{L^{2}\left(D_{k, i}\right)} .
$$

In the remainder, we shall tacitly assume that the coarse mesh is fine enough for $P_{k, i}$ to exist and satisfy (4.6) for smoothing subspaces on all refinements.

A well known technique for verifying (4.4) for the Gauss-Seidel (or Jacobi) smoother is by combining the above stability estimate with a perturbation argument. To apply this technique, we need the next lemma $[3,7,11]$. The mesh $\mathcal{T}_{1}$ is fixed and determines the 
angles of all triangles in all of the refined meshes. Thus, all meshes are quasi-uniform with constants of uniformity that are independent of mesh level. Consequently the smoothing subspaces $V_{k, i}$ satisfy the so-called limited interaction property, i.e., for every $k$ and $i$, the number of domains $D_{k, \ell}$ such that meas $\left(D_{k, i} \cap D_{k, \ell}\right)>0$ is uniformly bounded by some constant $\ell_{0}$ independent of $J$.

Lemma 4.2 (Perturbation for smoothers). If there is a constant $C_{0}>0$ such that the operators $Z_{k, i}=P_{k, i}-\hat{P}_{k, i}$ satisfy

$$
\left\|Z_{k, i}\right\|_{\Lambda} \leq C_{0} h_{k}
$$

for all $k$ and $i$, then there is a constant $c$ depending only on the limited interaction constant $\ell_{0}$ such that the estimate (4.4) holds for $Z_{k}$ with $C_{1}=c C_{0}$ whenever $h_{1} \leq h_{0}$ for $h_{0}$ sufficiently small.

With these preparations, we are able to prove a theorem on the convergence of Algorithm 3.2 applied to the complex coefficient problem (1.2).

Theorem 4.1. There exist constants $C_{*}>0$ and $H>0$ such that whenever the coarsest mesh size $h_{k_{0}}$ is less than $H$,

$$
\|\mathcal{E}-\hat{\mathcal{E}}\|_{\Lambda} \leq C_{*} h_{k_{0}}^{s / 2}
$$

The constants $C_{*}$ and $H$ are independent of $J$, the number of refinement levels in the multigrid algorithm.

Remark 4.1. One might consider the multigrid algorithm applied to $\hat{a}(\cdot, \cdot)$ as a "classical" or "textbook" multigrid application $[2,7,8]$. The above theorem shows that convergence rate for multigrid applied to the complex coefficient problem tends to the convergence rate for the classical multigrid application. This behavior is also illustrated by the computational examples of the next section.

Proof of Theorem 4.1. By Lemmas 4.1 and 4.2, it suffices to verify (4.7) and (4.3) with $\epsilon=C h_{k_{0}}^{s / 2}$.

To verify (4.7), we start by defining the form

$$
d_{\kappa}(u, v)=\int_{\Omega}\left(\frac{\kappa-\alpha(x, y)}{\kappa}\right) \nabla u \cdot \overline{\nabla v} d x d y,
$$

where $\kappa$ is any complex constant. Then

$$
\kappa \hat{a}(u, v)-a(u, v)=\kappa d_{\kappa}(u, v) .
$$

Moreover, for any $v_{i}$ in $V_{k, i}$,

$$
\begin{aligned}
\kappa \hat{a}\left(Z_{k, i} u, v_{i}\right) & =\kappa \hat{a}\left(P_{k, i} u, v_{i}\right)-\kappa \hat{a}\left(\hat{P}_{k, i} u, v_{i}\right) \\
& =a\left(P_{k, i} u, v_{i}\right)+\kappa d_{\kappa}\left(P_{k, i} u, v_{i}\right)-\kappa \hat{a}\left(\hat{P}_{k, i} u, v_{i}\right), \\
& =a\left(u, v_{i}\right)+\kappa d_{\kappa}\left(P_{k, i} u, v_{i}\right)-\kappa \hat{a}\left(u, v_{i}\right), \\
& =-\kappa d_{\kappa}\left(u, v_{i}\right)+\kappa d_{\kappa}\left(P_{k, i} u, v_{i}\right) .
\end{aligned}
$$

Thus,

$$
\hat{a}\left(Z_{k, i} u, v_{i}\right)=d_{\kappa}\left(P_{k, i} u-u, v_{i}\right) \quad \text { for all } v_{i} \in V_{k, i} \text {. }
$$


Now, if we choose $\kappa=\alpha_{i}:=\alpha\left(x_{k, i}\right)$, using the fact that the support of functions in $V_{k, i}$ extend only a distance $O\left(h_{k}\right)$, we find that for any $v_{i}$ in $V_{k, i}$,

$$
\begin{aligned}
\left|d_{\alpha_{i}}\left(u, v_{i}\right)\right| & =\left|\int_{L^{2}\left(D_{k, i}\right)}\left(\frac{\alpha_{i}-\alpha(x, y)}{\alpha_{i}}\right) \nabla u \cdot \nabla v_{i} d x d y\right| \\
& \leq C h_{k} \frac{\|\nabla \alpha\|_{L^{\infty}\left(D_{k, i}\right)}}{\left|\alpha_{i}\right|}\|\nabla u\|_{L^{2}\left(D_{k, i}\right)}\left\|v_{i}\right\|_{\Lambda} .
\end{aligned}
$$

Using this in (4.9), we can finish the proof of (4.7):

$$
\begin{aligned}
\left\|Z_{k, i}\right\|_{\Lambda} & =\sup _{u \in V_{k}, v_{i} \in V_{k, i}} \frac{\hat{a}\left(Z_{k, i} u, v_{i}\right)}{\|u\|_{\Lambda}\left\|v_{i}\right\|_{\Lambda}} \\
& \leq C h_{k}\left\|I-P_{k, i}\right\|_{\Lambda} \leq C h_{k} .
\end{aligned}
$$

where we have used (4.6). This proves (4.7).

It now only remains to prove $(4.3)$ for $Z_{1}=P_{1}-\hat{P}_{1}$. There is an identity analogous to $(4.9)$ for $Z_{1}$ :

$$
\hat{a}\left(Z_{1} u, v_{1}\right)=d_{\kappa}\left(P_{1} u-u, v_{1}\right) \text { for all } v_{1} \in V_{1} .
$$

Its proof follows from (4.8) along the lines of the derivation of (4.9). Now, let $\left\{\phi_{i}: i=\right.$ $\left.1, \ldots N_{1}\right\}$ be the nodal basis for $V_{1}$. Clearly, $\phi_{i}$ is in $V_{1, i}$. Then, for any $u \in V_{J}$, expanding

$$
Z_{1} u=\sum_{i=1}^{N_{1}} c_{i} \phi_{i}
$$

we have, by (4.11),

$$
\left\|Z_{1} u\right\|_{\Lambda}^{2}=\sum_{i=1}^{N_{1}} \bar{c}_{i} \hat{a}\left(Z_{1} u, \phi_{i}\right)=\sum_{i=1}^{N_{1}} \bar{c}_{i} d_{\alpha_{i}}\left(P_{1} u-u, \phi_{i}\right)
$$

where $\alpha_{i}$, as before, equals $\alpha\left(x_{1, i}\right)$. Now, using (4.10), and an inverse inequality,

$$
\begin{aligned}
\left\|Z_{1} u\right\|_{\Lambda}^{2} & \leq C \sum_{i=1}^{N_{1}}\left|\bar{c}_{i}\right| h_{1}\left\|\nabla\left(P_{1} u-u\right)\right\|_{L^{2}\left(D_{k, i}\right)}\left\|\nabla \phi_{i}\right\|_{L^{2}\left(D_{k, i}\right)} \\
& \leq C \sum_{i=1}^{N_{1}}\left|\bar{c}_{i}\right|\left\|\phi_{i}\right\|_{L^{2}\left(D_{k, i}\right)}\left\|\nabla\left(P_{1} u-u\right)\right\|_{L^{2}\left(D_{k, i}\right)} \\
& \leq C\left(\sum_{i=1}^{N_{1}}\left|c_{i}\right|^{2}\left\|\phi_{i}\right\|_{L^{2}\left(D_{k, i}\right)}^{2}\right)^{1 / 2}\left(\sum_{i=1}^{N_{1}}\left\|\nabla\left(P_{1} u-u\right)\right\|_{L^{2}\left(D_{k, i}\right)}^{2}\right)^{1 / 2}
\end{aligned}
$$

Since $c_{i}$ are the coefficients of $Z_{1} u$, by quasiuniformity, the term inside the first parenthesis is bounded by $C\left\|Z_{1} u\right\|_{\Omega}$. The term in the second parenthesis can be controlled by the limited interaction property, so we obtain

$$
\left\|Z_{1} u\right\|_{\Lambda}^{2} \leq C\left\|Z_{1} u\right\|\left\|P_{1} u-u\right\|_{\Lambda} \leq C\left\|Z_{1} u\right\|\|u\|_{\Lambda} .
$$

We used Theorem 2.1 for the last inequality above. 
Now, by the finite element duality argument applied to the forms $\hat{a}(\cdot, \cdot)$ and $a(\cdot, \cdot)$ (cf. proof of (2.17) in Theorem 2.1),

$$
\begin{aligned}
& \left\|\hat{P}_{1} u-u\right\| \leq C h^{s}\left\|\hat{P}_{1} u-u\right\|_{\Lambda}, \\
& \left\|P_{1} u-u\right\| \leq C h^{s}\left\|P_{1} u-u\right\|_{\Lambda} .
\end{aligned}
$$

Hence, Theorem 2.1 and the obvious stability property of $\hat{P}_{1}$ give

$$
\left\|Z_{1} u\right\| \leq\left\|\left(P_{1} u-u\right)\right\|+\left\|\left(u-\hat{P}_{1} u\right)\right\| \leq C h^{s}\|u\|_{\Lambda} .
$$

This together with (4.12) gives (4.3).

Remark 4.2. The techniques of the above proof immediately generalize to the $\mathrm{V}$-cycle multigrid algorithm $\mathrm{MG}_{J}(\cdot, \cdot)$. Note that the corresponding algorithm for $\hat{a}(\cdot, \cdot)$ results in a symmetric error reduction operator in the $\Lambda$-inner product even with smoothers based on Gauss-Seidel. This is a consequence of the use of $R_{k}^{\prime}$ in the third step. This seems to be a natural strategy as the problem corresponding to $\hat{a}(\cdot, \cdot)$ on $V_{J}$ is symmetric and positive definite.

\section{NUMERICAL EXPERIMENTS}

In this section, we report the results of numerical experiments illustrating the convergence of the multigrid algorithm on the model problem (1.1). We consider two examples for the complex coefficient $\alpha$ in (1.1) and report the results for each.

In both cases, the domain $\Omega$ is taken to be the unit square. The coarse mesh $\mathcal{T}_{1}$ consists of triangles obtained by dividing $\Omega$ into $4 \times 4$ congruent squares and connecting the positively sloped diagonals. We obtain multilevel meshes $\mathcal{T}_{k}$ as described previously and define $h_{k}$ to be the distance between two adjacent mesh points of $\mathcal{T}_{k}$ on any horizontal or vertical line. To iterate for the solution to (3.1), we apply V-cycle iterations, i.e., we apply (3.3) with Algorithm 3.2 using the point Gauss-Seidel smoother based on the lexicographical like ordering and an arbitrary initial iterate to solve the system $A_{J} u=$ 0 . As the error reduction operator for the process is linear, this is the same as taking $f=-A_{J} u_{0}$ with a zero starting iterate. We computed the stiffness matrices at all levels using the mid point quadrature rule applied at the finest grid.

To report the results, we start with our first example of $\alpha$, namely

$$
\alpha(x, y)=1+\hat{\imath} K \sin (\pi(2 y-1) / 2)
$$

where $K$ is a real constant and $\hat{\imath}$ denotes the imaginary unit. The specific form of the coefficient in (5.1) falls into the category of coefficients in Example 1.1, hence (1.3) and (2.1) hold. Therefore, from Theorem 4.1, we find that if we choose the coarse mesh in the multigrid algorithm sufficiently fine then the $\mathrm{V}$-cycle convergence rate is bounded independently of the mesh size.

The results that we obtain for $k_{0}=1$ (in other words, with the coarse mesh size $\left.h_{c}=1 / 4\right)$ and $K=0,1,20,100$ are given in Table 5.1 where we report the number of iterations required to reduce the $\Lambda$-norm of the error by a factor of $10^{-5}$. The appearance of a star $(\star)$ indicates that the algorithm failed to converge. The divergence in the case of $K=100$ clearly indicates that the coarse grid is not fine enough for this coefficient. Note that $K=0$ corresponds to multigrid algorithm applied to $\hat{A}$ and results in the reduction operator $\hat{\mathcal{E}}$. The theory developed in the earlier sections shows that $\mathcal{E}$ converges to $\hat{\mathcal{E}}$ as the coarse grid becomes finer. This is seen for $K=1$ as the corresponding iteration 


\begin{tabular}{|c|cccc|}
\hline$h_{c}=1 / 4$ & $\begin{array}{c}\text { V-cycles } \\
(K=0)\end{array}$ & $\begin{array}{c}\text { V-cycles } \\
(K=1)\end{array}$ & $\begin{array}{c}\text { V-cycles } \\
(K=20)\end{array}$ & $\begin{array}{c}\text { V-cycles } \\
(K=100)\end{array}$ \\
\cline { 1 - 4 }$h_{J}$ & 7 & 7 & 20 & $\star$ \\
$1 / 8$ & 7 & 7 & 15 & $\star$ \\
$1 / 16$ & 7 & 7 & 13 & $\star$ \\
$1 / 64$ & 7 & 7 & 12 & $\star$ \\
$1 / 128$ & 7 & 7 & 12 & $\star$ \\
\hline
\end{tabular}

TABLE 5.1. Iteration counts as a function of $K$ and $h_{J}$ for $k_{0}=1\left(\right.$ or $\left.h_{c}=1 / 4\right)$.

\begin{tabular}{|c|ccccc|}
\hline$h_{J}=1 / 256$ & $\begin{array}{c}\text { V-cycles } \\
(K=0)\end{array}$ & $\begin{array}{c}\text { V-cycles } \\
(K=1)\end{array}$ & $\begin{array}{c}\text { V-cycles } \\
(K=20)\end{array}$ & $\begin{array}{c}\text { V-cycles } \\
(K=100)\end{array}$ \\
\hline$h_{c}$ & 7 & 7 & 11 & $\star$ \\
$1 / 4$ & 7 & 7 & 9 & $\star$ \\
$1 / 8$ & 7 & 7 & 8 & 16 \\
$1 / 32$ & 7 & 7 & 7 & 10 \\
$1 / 64$ & 7 & 7 & 7 & 8 \\
$1 / 128$ & 7 & 7 & 7 & 7 \\
\hline
\end{tabular}

TABLE 5.2. Iteration counts as a function of $K$ and $h_{c}$ for $h_{J}=1 / 256$.

numbers already coincide with those for $K=0$. The number of iterations for $K=20$ decrease as the fine grid mesh size becomes smaller. Of course, the iteration counts for $K=20$ need not converge to that of $K=0$ unless the coarse grid is fine enough.

To see the dependence on the coarse grid size, we fixed the fine grid mesh size at $h_{J}=1 / 256$ and varied $k_{0}$ (or the coarse mesh size $h_{c}$ ). The results are in Table 5.2. Again, we see that the iteration numbers for $K=1$ are the same as the $K=0$ case, while those for $K=20$ are clearly converging to those of $K=0$ as $h_{c}$ becomes small. The case of $K=100$ remains unstable for coarse grid mesh sizes up to $1 / 8$ but converges for $1 / 16$. Again, we see the iteration numbers tending to those of $K=0$, as expected from the theory.

By considering large values of $K$ in (5.1), we found the condition that the coarse grid be sufficiently fine is unavoidable in practice when using Algorithm 3.2. Hence, it seems that a theoretical convergence proof without this condition is not possible for general complex coefficients. Such behavior of multigrid algorithms is well known when applied to the Helmholtz equation [11]. As the wave number in the lower order term of the Helmholtz equation increases, the coarse grid must be made progressively finer to "resolve the wave". Our study shows that this behavior is not restricted to the stationary wave equation but can also arise when a coefficient is complex, even in the absence of a negative lower order term.

Finally, we present our second example to illustrate that the proposed multigrid algorithms work even when the problem is not coercive in the sense of Example 1.1. Specifically, we consider the coefficient given by

$$
\alpha(x, y)=(1-r)^{2}+r^{4} \exp (4 \hat{\imath} \theta)
$$




\begin{tabular}{|c|c|c|c|c|}
\hline$h_{J}$ & $\begin{array}{c}\text { V-cycles } \\
\left(h_{c}=1 / 4\right)\end{array}$ & $\begin{array}{c}\text { V-cycles } \\
\left(h_{c}=1 / 32\right)\end{array}$ & $\begin{array}{c}\text { V-cycles } \\
\left(h_{c}=1 / 64\right)\end{array}$ & $\begin{array}{c}\text { V-cycles } \\
\left(h_{c}=1 / 128\right)\end{array}$ \\
\hline $1 / 64$ & $\star$ & $\star$ & & \\
\hline $1 / 128$ & $\star$ & $\star$ & 43 & \\
\hline $1 / 256$ & $\star$ & $\star$ & 7 & 7 \\
\hline $1 / 512$ & $\star$ & $\star$ & 7 & 7 \\
\hline
\end{tabular}

TABLE 5.3. Iteration counts for (5.2) and Gauss-Seidel (lexicographical ordering).

\begin{tabular}{|c|c|c|c|c|}
\hline$h_{J}$ & $\begin{array}{c}\text { V-cycles } \\
\left(h_{c}=1 / 4\right)\end{array}$ & $\begin{array}{c}\text { V-cycles } \\
\left(h_{c}=1 / 8\right)\end{array}$ & $\begin{array}{c}\text { V-cycles } \\
\left(h_{c}=1 / 64\right)\end{array}$ & $\begin{array}{c}\text { V-cycles } \\
\left(h_{c}=1 / 128\right)\end{array}$ \\
\hline $1 / 64$ & 26 & 24 & & \\
\hline $1 / 128$ & 27 & 24 & 19 & \\
\hline $1 / 256$ & 27 & 24 & 20 & 19 \\
\hline $1 / 512$ & 25 & 23 & 20 & 20 \\
\hline
\end{tabular}

TABLE 5.4. Iteration counts for (5.2) and damped Jacobi.

where $r, \theta$ are the polar coordinates of $(x, y)$. Even though we cannot show that this problem satisfies (1.3), we shall compute with it anyway (of course, (2.1) holds). The iteration numbers for this problem are given in Table 5.3 as a function of the coarse and fine grid sizes used in the multigrid algorithm. (The algorithm and parameters used to obtain Table 5.3 are the same as in the first example of $\alpha$.)

It turned out that convergence was particularly troublesome for this problem with these settings. As seen from Table 5.3, we had to use a coarse grid of mesh size $h_{c}=1 / 64$ to get a convergent multigrid algorithm. In accordance with the theory, the number of iterations tends to that for $\hat{A}$ once the coarse grid mesh size is small enough to obtain convergence. We did not anticipate needing such small coarse grid mesh sizes.

Further experiments revealed that if we change the smoother from the Gauss-Seidel smoother with lexicographical ordering to Gauss-Seidel with the red-black ordering, we obtained convergence with a mesh size as coarse as $h_{c}=1 / 4$. Thus, the lexicographical ordering seems to be not very stable for the Gauss-Seidel iteration applied to this problem. Also, we obtained convergence for any coarse grid with damped Jacobi smoothing with a damping factor of $1 / 2$ (see Table 5.4). In this case, the iteration numbers for $\hat{\mathcal{E}}$ with this smoother and $h_{c}=1 / 16$ were 19,20,20, 20 for fine grids of size $1 / 32,1 / 64,1 / 128$ and $1 / 256$, respectively. Again, as suggested by the theory, we see the iteration numbers for

$\mathcal{E}$ converging to those of $\hat{\mathcal{E}}$ for small $h_{c}$. We made no attempt at optimizing the damping factor so, quite possibly, better error counts could be achieved with other damping factors.

\section{REFERENCES}

[1] R. BANK, A comparison of two multilevel iterative methods for nonsymmetric and indefinite elliptic finite element equations, SIAM J. Numer. Anal., 18 (1981), pp. 724-743.

[2] R. E. Bank And T. Dupont, An optimal order process for solving finite element equations, Math. Comp., 36 (1981), pp. 35-51. 
[3] J. H. Bramble, D. Y. Kwak, and J. E. Pasciak, Uniform convergence of multigrid V-cycle iterations for indefinite and nonsymmetric problems, SIAM J. Numer. Anal., 31 (1994), pp. 17461763.

[4] J. H. BRamble And J. E. PAsciak, Analysis of a finite PML approximation for the three dimensional time-harmonic Maxwell and acoustic scattering problems, Math. Comp., 76 (2007), pp. 597614 (electronic).

[5] — Analysis of a finite element PML approximation for the three dimensional time-harmonic Maxwell problem, Math. Comp., 77 (2008), pp. 1-10 (electronic).

[6] J. H. Bramble, J. E. Pasciak, J. P. Wang, And J. Xu, Convergence estimates for product iterative methods with applications to domain decomposition, Math. Comp., 57 (1991), pp. 1-21.

[7] J. H. BRAmble AND X. Zhang, The analysis of multigrid methods, in Handbook of numerical analysis, Vol. VII, North-Holland, Amsterdam, 2000, pp. 173-415.

[8] A. Brandt, Multi-level adaptive solutions to boundary-valued problems, Math. Comp., 31 (1977), pp. 333-390.

[9] F. Brezzi and M. Fortin, Mixed and Hybrid Finite Element Methods, no. 15 in Springer Series in Computational Mathematics, Springer-Verlag, New York, 1991.

[10] G. B. Folland, Introduction to Partial Differential Equations, Princeton University Press, Princeton, New Jersey, 1995.

[11] J. Gopalakrishnan, J. E. Pasciak, And L. F. Demkowicz, Analysis of a multigrid algorithm for time harmonic Maxwell equations, SIAM J. Numer. Anal., 42 (2004), pp. 90-108 (electronic).

[12] P. Grisvard, Elliptic Problems in Nonsmooth Domains, no. 24 in Monographs and Studies in Mathematics, Pitman Advanced Publishing Program, Marshfield, Massachusetts, 1985.

[13] S. KIM AND J. E. PASCIAK, The computation of resonances in open systems using a perfectly matched layer. Submitted to Math. Comp.

[14] J. NEČAs, Les méthodes directes en théorie des équations elliptiques, Masson et C ${ }^{\mathrm{ie}}$, Éditeurs, Paris, 1967.

[15] J. PeEtre, Espaces d'interpolation et théorème de Soboleff, Ann. Inst. Fourier (Grenoble), 16 (1966), pp. $279-317$.

[16] A. H. Schatz, An observation concerning Ritz-Galerkin methods with indefinite bilinear forms, Math. Comp., 28 (1974), pp. 959-962.

[17] L. TARTAR, Topics in nonlinear analysis, vol. 13 of Publications Mathématiques d'Orsay 78, Université de Paris-Sud Département de Mathématique, Orsay, 1978.

[18] J. Xu, A new class of iterative methods for nonselfadjoint or indefinite problems, SIAM J. Numer. Anal., 29 (1992), pp. 303-319.

Department of mathematics, University of Florida, Gainesville, FL 32611-8105.

E-mail address: jayg@math.ufl.edu

Department of Mathematics, Texas A\&M University, College Station, TX $77843-3368$.

E-mail address: pasciak@math.tamu.edu 